\title{
On the Potential of Web Services in Network Management
}

\author{
ZiHeng Liu ${ }^{1}, Y u \mathrm{Bai}^{2}$, YouQing Wan ${ }^{3}$ \\ 1 The Department of Information Techonlogy, HuaZhong Normal \\ University; Wuhan, China,lzh20201@yahoo.com.cn \\ 2 The Department of Information Management, HuaZhong Normal \\ University; Wuhan, China,baiyu926@sohu.com \\ 3 The Deaprtment of Management, WuHan University of \\ Technology,youqingwan123@sohu.com
}

\begin{abstract}
These days, Web services, which are based on XML and consist of several XML-related technologies, have been emerging as a promising technology, and it seems that Web services may be used in the field of network management. In this paper, we examine Web services as a XML-based approach to network management. Since Web services are a Service-Oriented Architecture (SOA) more than just a set of technologies, it could be used in XML-based network management, not only at the technology level, but also at the architecture level, which has many remarkable advantages and may be more effective in network management along with the concepts of Peer-toPeer and Point-to-Point, in order to make a better use of its capability. Correspondingly, two models are presented to further demonstrate the potential of Web services in XML-based network management.
\end{abstract}

\section{Introduction}

Since network management research and standardization started in the late $1980 \mathrm{~s}$, there still has been no such a framework and technology that satisfies the general needs of network management. Recently, more attention is paid to XML-based approaches, and in particular, as a standard based on XML, Web services seem to be appropriate for network management.

Some organizations have participated in the research of using Web services in network management, such as the Organization for the Advancement of Structured Information Standards (OASIS) and the Network Management Research Group (NMRG) of the Internet Research Task Force (IRTF). In addition, several researchers have also studied the standardizations and prototypes for applying Web

Please use the following format when citing this chapter:

Liu, Z., Bai, Y., Wan, Y., 2007, in IFIP International Federation for Inlomation Processing, Volume 252, Integration and Innovation Orient to F-Society Volume 2, eds. Wang, W., (Boston: Springer), pp. 81-87. 
services to network management [1], [2]. But only few investigators have ever considered its potential in management [3].

In most studies, the real potential of Web services in XML-based network management has rarely been discussed. However, as a XML-based standardization, Web services have many advantages offered by XML and its related technologies, all of which facilitate its use in XML-based network management. What is more, since Web services is a Service-Oriented Architecture (SOA) more than a set of technology, a study at the architecture level may seem more effective than just at the technology level, in order to make full use of its capability. The aim of this paper is then to provide the advantages of Web services in network management from XML's point of view, and based on the current development of XML-based network management, to apply Web services at the architecture level more than just at the technology level.

The organization of this paper is as follows. First, a brief introduction of XML and Web services will be offered in Section 2, with an emphasis on the architecture. Section 3 will describe the advantages of Web services in network management, focusing on the benefits offered by XML-related technologies, including Web Services Description Language (WSDL) and Simple Object Access Protocol (SOAP). Sequentially in Section 4, we will discuss XML-based network management using Web services not only at the technology level but also at the architecture level, and two corresponding models will then be given. In addition, the benefits from the latter one are analyzed in detail. Finally, we conclude our work and discuss future work in Section 5 .

\section{Preliminaries}

Nowadays, generic Internet technologies such as XML are being adopted to manage network resources. Thus in this background, XML-based network management, which applies XML technologies to network management, has been regarded as an alternative to existing network management. Extensible Markup Language (XML) is a meta-markup language standardized by the World Wide Web Consortium (W3C) for document exchange in the web. XML is now a standard that is supported and accepted by thousands of vendors as well as a lot of related technologies and tools.

Under this background, Web services have also been emerging as a promising Internet-oriented technology and architecture for network management [4]. A number of widely adopted Web services technologies are now available, such as Simple Object Access Protocol (SOAP) [5] [6] [7], Web Services Description [8] [9] [10] [11], and Universal Description Discovery, and Integration (UDDI) [12].

The word "services" in Web services refers to a Service-Oriented Architecture (SOA). In an SOA, functionality is "published" on a network where two important capabilities are also provided - "discovery", the ability to find the functionality, and "binding", the ability to connect the functionality. So when considering a SOA, these three parts must be take into account, which are briefly presented as "publish", "find", and "bind".

The concept of a SOA is not new, for service-oriented architectures have been used for years. However, what is relatively new is the emergence of Web services- 
based SOAs. In a Web services-based SOA, three important roles are Web service provider, Web service requester, and Web service register, which correspond to the "publish", "find", and "bind" aspects of a SOA.

\section{The advantage of Web service in XML-based network management}

As one of the emerging standards based upon XML, Web services are a generic technology and the use of standard XML protocols or technologies makes Web services platform-, programming language-, and vendor-independent. The support of XML technologies, such as WSDL and SOAP, provides the capability for the standardizations of management information definition and access, which are very important in network management.

\subsection{Standardization of management information definition by WSDL}

A Web service is described in a WSDL document. In order to easily use Web services for network management, standardization of management information definition is needed. On the other hand, with the mechanism provided by WSDL to describe a Web service in a modular manner using the elements <import> and <include>, modularization can be achieved.

With regard to the division of WSDL documents, some attempts have been done. The Universal Description, Description, Integration (UDDI) Technical Committee recommends "two separate WSDL definitions" containing an interface part and an implementation part. With WSDL import mechanism, Reference [1] suggests "three separate WSDL definitions" containing an abstract part:

- messages and interfaces (the what part) and two concrete parts:

- a binding (the how part)

- a service (the where part)

In our study, we propose the latter one, for it seems more reasonable according to the functionalities of each element.

\subsection{Standardization of management information access by SOAP over HTTP}

According to related specifications, SOAP focuses on the basic forms of transporting messages, regardless of the transfer protocol. Since SOAP messages and transfer protocols are strictly separated from each other, SOAP has the capability of combining with any transfer protocol. Considering the common usability and practicability, and the fact that most applications are transmitted through HTTP, the combination of SOAP messages and HTTP is undoubtedly the most practical and widely used means to implement the interconnections between services.

Thus in this way, SOAP over HTTP, which supports its own Remote Procedure Calls (RPC) interfaces, becomes a natural application protocol for network management and this default transport scheme provides a standardization of management information access. 


\section{XML-based network management using Web services}

XML-based network management systems have become more and more popular these days, for it applies XML technologies to network management. However, these systems differ much in the extent of using the XML technologies. Most of these systems just use a few simple XML technologies, while some do make a better use of the XML technologies, such as the system presented in [13].

As to current attempts to use Web services in XML-based network management, the usage level of Web services must be taken into account. Since Web services are a SOA more than a set of XML-based technologies, it is reasonable to distinguish the XML-based network management using Web services at two levels: the technology level and the architecture level. Recent studies focus more on the technology level, but in order to make a better use of Web services-based SOA, it seems to be more appropriate to focus on the architecture level.

\subsection{At the technology level}

Web services technologies, such as WSDL and SOAP over HTTP, are now used in XML-based network management. Using them carefully can provide high-level operations to deal with complicated management problems, such as configuration management and device management, etc. Figure 1 illustrates a manager-agent model of XML-based network management using Web services at the technology level

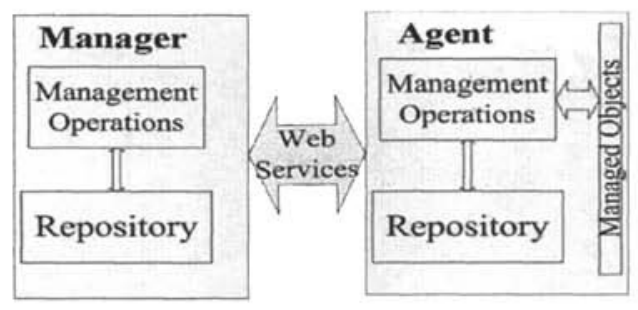

Figure 1. A common model at the technology level

As is shown in Figure 1, either the manager or the agent is composed of two modules: Management Operations, and a Repository, which contains RPC operation WSDL document. The Web Services module usually contains the SOAP engine, including the HTTP server/client, which provides SOAP APIs for initiating a bidirectional connection. The manager can access the Web service on the agent through SOAP over HTTP (or another lower network protocol), which enables the system to support network management. After the manager encapsulates the request message with SOAP, it is sent to the agent through HTTP. When receiving the request, the agent parses it, executes the corresponding service and returns the result to the manager still with SOAP over HTTP. 


\subsection{At the architecture level}

Figure 1 just extends the manager-agent model for network management by the means of Web services technologies. However, in a distributed environment, or more exactly over a network, a number of managers and agents exist.

Bear in mind that, SOA and Web services are not just abstract concepts, but are real approaches to solving network management problems. A Web services-based SOA is essentially a collection of services. These services communicate with each other. The communication can involve either simple data passing or it could involve two or more services coordinating some activity, when some means of connecting services to each other is needed. Thus in this case, we could make a better use of the Web services-based SOA.

As a seamless integration of XML-based network management and Web services-based SOA, Figure 2 illustrates a common model at the architecture level, in which each entity can act as either an agent role ( $a$ Web service provider) or a manager role ( $a$ Web service requester), or both.
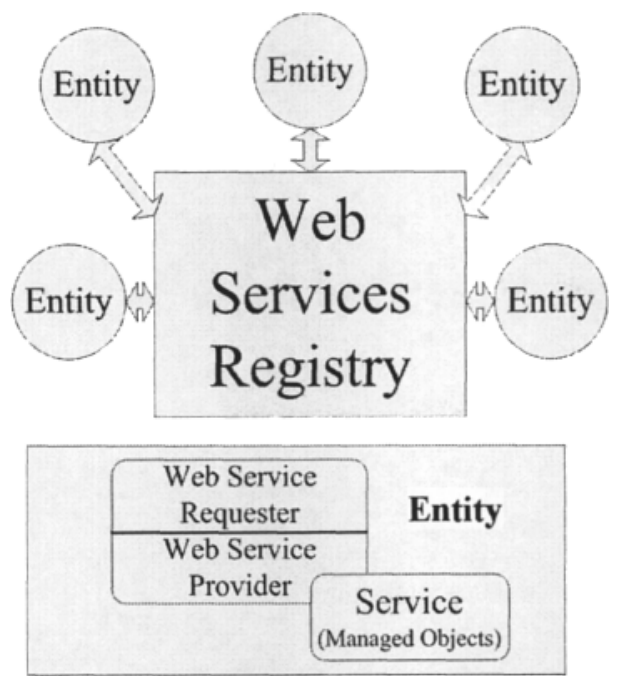

Figure 2. A common model at the architecture level

As is shown in Figure 2, two main components in the model are the Entity and, the Web Services Registry, which can be a private one for a particular network management task. The use of entities based on the Web services-based SOA, in fact, utilizes the concepts of Peer-to-Peer and Point-to-Point.

What makes peer-to-peer systems interesting is that they are totally distributed and all nodes are symmetric. On the other hand, point-to-point networks consist of many connections between individual pairs of machines, in contrast to broadcast networks. The concepts of Peer-to-Peer and Point-to-Point are essentially the same: the former one demonstrates the communication style, while the latter one reveals the link means. With the very use of these two concepts, the working flow of this model is as follows. 
First of all, these entities, each of which acts as a Web service provider in this model, register their own services of some managed objects to the Web Services Registry. When another entity, acting as a Web service requester, wants to acquire the information of one or more managed objects provided by one entity, it just needs to connect the Web Services Registry to get the access information to that entity, the procedure of which is based on the Web services and the peer-to-peer communication. To accomplish the point-to-point link, the entity can acquire the service through SOAP over HTTP or another transfer protocol.

In the following, the main advantages of this model will be discussed in detail.

First of all, a management process in OSI-SM architecture can be configured to act in either the agent role or the manager role, or both roles. However, in SNMP network management systems and existing XML-based network management systems, both of which have been widely used, a management process can only be configured to act in either role but never both. Therefore, the use of Web services can bring XML-based network management closer to OSI-SM, which is so far the strongest network management architecture that supports all the essential features in any management framework.

Additionally, Web services-based SOA can largely and effectively improve the current network management systems. On the one hand, one primary feature of Web services-based SOA is scalability, which can properly solve this problem in network management. Since services in an SOA are loosely coupled, applications that use these services tend to scale easily and certainly, more easily than applications in a tightly coupled environment. On the other hand, the flexibility of Web servicesbased SOA, which is provided by the loosely coupled, document-based, asynchronous nature of services, allows applications to be flexible, and easy to evolve with changing network management requirements.

\section{Conclusions and further work}

This paper discusses issues on the potential of Web services in the field of network management, and provides two models of XML-based network management using Web services at the technology level as well as at the architecture level, focusing more on the latter one, which has several remarkable advantages and makes a better use of Web services recur to the concepts of Peer-to-Peer and Point-to-Point.

Our study indicates that in XML-based network management, using Web services at the architecture level would be better than that at the technology level.

Further work is needed to implement XML-based network management systems using Web services at these two different levels, and performance tests are also needed in order to validate our point of view. In addition, considering the capability of Web services in coordinating network management, we also plan to design and implement a practical XML-based collaborative network management system in the future. 


\section{References}

1. J. Sloten, A. Pras, M. Sinderen, "On the Standardisation of Web service management operations", Proc. 10th Open European Summer School (EUNICE 2004) and IFIP WG 6.3 Workshop (June 2004), pp. 143-150.

2. T. Drevers, R. Meent, A. Pras, "Prototyping Web Services based Network Monitoring", Proc. 10th Open European Summer School (EUNICE 2004) and IFIP WG 6.3 Workshop(June 2004), pp. 135-142.

3. G. Pavlou, P. Flegkas, S. Gouveris, A. Liotta, "On Management Technologies and the Potential of Web Services", IEEE Communication Magazine(July 2004), pp. 58-66.

4. J. Schonwalder, A. Pras, J.P. Martin-Flatin, "On the Future of Internet Management Technologies", IEEE Communications Magazine(October 2003), pp. 90-97.

5. W3C, "SOAP Version 1.2 Part 0: Primer", W3C Recommendation, April 2007.

6. W3C, "SOAP Version 1.2 Part 1: Message Framework", W3C Recommendation(April 2007).

7. W3C, "SOAP Version 1.2 Part 2: Adjuncts", W3C Recommendation(April 2007).

8. W3C, "Web Services Description Language (WSDL) Version 2.0 Part 0: Primer", W3C Proposed Recommendation(May 2007).

9. W3C, "Web Services Description Language (WSDL) Version 2.0 Part 1: Core Language", W3C Proposed Recommendation(May 2007).

10. W3C, "Web Services Description Language (WSDL) Version 2.0 Part 2: Message Exchange Patterns", W3C Working Draf(March 2004).

11. W3C, "Web Services Description Language (WSDL) Version 2.0 Part 3: Bindings", W3C Working Draf(August 2004).

12. UDDI Spec TC, "UDDI Version 3.0.2 UDDI Spec Technical Committee Draft", UDDI Spec Technical Committee Draft( (October 2004).

13. M. Choi, W. Hong, H. Ju, "XML-Based Network Management for IP Network", ETRI Journal, Volume 25(Number 6, December 2003), pp. 445-463. 